\title{
INTERVENCIONES POLITICAS DE PRIMER ORDEN: el caso de la política chilena de la transparencia
}

\section{INTERVENÇÕES POLÍTICAS DE PRIMEIRA ORDEM: o caso da política chilena de transparência}

\author{
Patricio Valdivieso* \\ Arturo Vallejos-Romero ${ }^{* *}$
}

\begin{abstract}
Este estudio evalúa e interpreta las dificultades que ha encontrado la política chilena de transparencia en los municipios con el apoyo de la teoría de los sistemas sociales. Argumenta que, en el contexto latinoamericano actual, las políticas públicas deben dar respuesta a una sociedad crecientemente compleja y diversificada, con una aproximación de persuasión, dialogo y facilitación. Explica que la política de transparencia, en cuanto intervención de primer orden, ha sido una política de comando y control, y que en su reformulación debiese considerar lo que entienden por transparencia los ciudadanos y los funcionarios municipales.

PALABRAS-CLAVE: Política pública. Intervención de segundo orden. Transparencia
\end{abstract}

\section{INTRODUCCION}

Este estudio evalúa e interpreta las dificultades que ha encontrado la política chilena de transparencia en los municipios, con el apoyo de la teoría de los sistemas sociales desarrollada en la sociología, principalmente por Niklaus Luhman (1997) y en la ciencia política por David Easton (1965).

En una parte de la literatura, la transparencia es definida como la apertura de las instituciones del Estado hacia la mirada y el escrutinio público (Florini 2002; Piotrowski et al. 2010). Por lo tanto, las políticas de transparencia consisten en facilitar el acceso a la información sobre la legislación, los

* Doctor Philosopie. Profesor Titular de la Universidad de los Lagos, asociado a ORPAS de la Universidad Bernardo O'Higgins. Este artículo sintetiza resultados de investigación del marco teórico de la ejecución del proyecto FONDECYT 1140672, CONICYT/Chile. Calle Lord Cochranne 1056, Osorno, X Región de Los Lagos, Chile.pvaldivf@gmail.com

* * Doctor en Ciencias Sociales (Flacso-México). Profesor Asociado e Investigador de la Universidad de La Frontera, Chile. Su aporte en la parte teórica de la intervención de segundo orden de este artículo se deriva del proyecto FONDECYT 1120554

Avenida Francisco Salazar 01145, Casilla 54-D. Temuco - Chile. arturo.vallejos@ufrontera.cl procedimientos administrativos, los presupuestos y otros asuntos de interés público, y esa información es ordenada y dispuesta de forma tal que sea completa, clara y auditable (Heise 1985; OECD 1996, 2000, 2002; Rawlins 2009). En un sentido más amplio, la transparencia fortalecería la calidad de la gobernanza democrática, al incentivar la responsabilidad de los gobernantes, facilitar la participación y el control social (Piotrowski et al. 2010). En consecuencia, desde hace una década a esta parte, los gobiernos de América Latina han perfeccionado su legislación sobre transparencia, prescribiendo que las instituciones y los servicios públicos deben disponer de diversas informaciones en sus sitios web sobre la legislación, los procedimientos, la gestión, el personal y el presupuesto, entre otros.

Chile pertenece al grupo de países latinoamericanos que dispone de una política de transparencia e internacionalmente se ha comprometido a mejorar las prácticas en el sector público. El año 2008, el gobierno de la Presidenta Michelle Bachellet hizo aprobar una Ley de Transparencia, la cual dispone que los servicios públicos y 
municipios deben disponer de ciertos ítems de información en sus sitios web, y creó un Consejo para la Transparencia con el propósito de supervisar la política y fomentar una cultura de transparencia en el país. Pero, a tres años de ser aprobada la ley, pocos municipios disponían la información prescrita en sus sitios web, y aparentemente la política no ha tenido efectos en los niveles de participación y confianza pública. En las elecciones municipales del año 2012, la abstención llegó al 60\% (información disponible en www.servel.cl), y las encuestas de opinión continúan indicando bajos niveles de confianza social y en las instituciones.

En este estudio, argumentamos que el problema de la política chilena tiene relación con el origen y el enfoque de la misma, pues se trata de una intervención de primer orden, una política de comando y control, cuyo énfasis está puesto en qué tipo de información deben proveer los municipios en sus sitios web, sin consultar a los ciudadanos qué transparencia quieren, y sin considerar los contextos sociales y municipales, especialmente de los funcionarios públicos encargados de aplicar la política. Proponemos que, en una fase de reformulación, la política podría transitar hacia una intervención de segundo orden, con un mayor énfasis en la persuasión, un enfoque más dialogante y facilitador, que considere condiciones y factores que han quedado al margen. Con el apoyo de la teoría de los sistemas sociales, fundamentaremos por qué una intervención política de segundo orden es deseable, en el actual contexto latinoamericano y chileno, y con información sobre el caso de la política chilena de transparencia y sus dificultades en los municipios, propondremos que las debilidades radican precisamente en el enfoque y la aplicación de la política.

Este estudio está estructurado de la siguiente manera: primero, sintetiza percepciones sobre el contexto latinoamericano actual, caracterizado por sociedades que se van volviendo crecientemente complejas y diferenciadas, donde los paradigmas convencionales de las políticas públicas enfrentan desafíos difíciles de superar; segundo, desarrolla el argumento de la importancia de la persuasión y la comunicación de las políticas públicas en la actualidad; tercero, presenta los principales fundamentos de una aproximación de segundo orden y una visión sistémica de la política, como una clave para avanzar hacia políticas públicas más inclusivas y efectivas; cuarto, sintetiza las principales informaciones sobre las características y génesis de la política chilena de la transparencia; quinto, aborda las dificultades de esa política en los gobiernos locales, municipales; sexto, explica estas dificultades por tratarse de una política de primer orden, distanciada de una aproximación sistémica integral, de comando y control, prescriptiva y poco dialogante; séptimo, se refiere a aspectos del contexto municipal y comunal que han quedado al margen de la política, tales como las rigideces y problemas en los municipios, la cultura política, los partidos y las organizaciones sociales; octavo, sintetiza percepciones de una muestra representativa de funcionarios municipales; y concluye con las implicaciones y orientaciones para transitar hacia una política de segundo orden.

\section{UNA APROXIMACIÓN DE SEGUNDO ORDEN PARA LAS POLÍTICAS PÚBLICAS}

\section{Contexto latinoamericano}

En las últimas décadas, las sociedades latinoamericanas se han vuelto crecientemente complejas, por el impacto transformador de un conjunto de cambios y modernizaciones económicas, políticas y sociales, con efectos en los patrones de comportamiento de los individuos y grupos sociales. Los efectos simultáneos de la transición demográfica, la urbanización y la aglomeración, la expansión de los mercados, la modernización de las tecnologías, los transportes y las comunicaciones, y las reformas económicas (Banco Mundial, 2009; Valdivieso, 2009) han favorecido la apertura y mayor integración de los sistemas económicos en el sistema económico mundial, donde predominan las reglas y dinámicas 
de mercado, las economías de escala y la especialización del trabajo. En la mayor parte de los países latinoamericanos, el Estado ha redefinido sus funciones regulatorias y sus relaciones con la sociedad, dando mayor espacio a los mercados y a subsistemas con mayor capacidad de auto-regulación. En el plano político y de las instituciones, desde fines de la década de 1970, las democracias han pasado a ser los sistemas políticos dominantes en la región. En relación con todo lo anterior, los efectos combinados de la globalización de los mercados y las sociedades, la democratización y el mayor pluralismo social y cultural (Valdivieso, 2002) han producido sistemas sociales, económicos y políticos que, día a día, son más complejos. Por lo tanto, el contexto social demanda nuevas regulaciones y modalidades para hacer políticas públicas distintas a las que imperaron en el pasado, en todas las fases de la política (identificación y diagnostico, formulación de la agenda, implementación, monitoreo y reformulación).

En los países latinoamericanos, la modernidad tardía se manifiesta de una forma cada vez más radical en la especialización de los sistemas sociales, es decir, en la diferenciación sustancial de las esferas de la política, el derecho, la salud, entre muchas otras, así como en una alta contingencia y una creciente complejidad. De este modo, la modernidad confronta a los tomadores de decisiones con una gran encrucijada. Las lógicas de funcionamiento y prácticas institucionalizadas han perdido la capacidad de resolver muchos de los problemas que se presentan en la vida común y que afectan la coexistencia, no sólo por limitaciones técnicas, fiscales, de infraestructura o humanas, sino porque la sobre determinación de eventos, reflejada en una infinidad de demandas sociales insatisfechas, ha aumentado exponencialmente. En todos los países de América Latina, esta problemática queda de manifiesto por una recurrente falta de sintonía entre las inquietudes y demandas sociales, por una parte, y respuestas de las instituciones y las políticas públicas, por otra. Esta falta de sintonía representa un desafío que rebasa las capacidades de los gobiernos para encontrar soluciones que den respuestas satisfactorias a problemas que persisten en el tiempo, tales como son las desigualdades, la pobreza, las problemáticas de la salud o en la educación.

Las realidades consignadas justifican una reflexión crítica con respecto a los paradigmas convencionales dominantes de intervención en las políticas públicas. En efecto, si ante los rasgos manifiestos de la sociedad actual, las políticas públicas y las intervenciones una y otra vez no pueden rescatar ni expresar de buena forma de los múltiples intereses (interpretaciones de necesidades) y demandas sociales, cabe preguntar por la validez de las aproximaciones y los déficits de las modalidades y estrategias que la intervención asume.

En la actualidad, en numerosos lugares, las intervenciones sociales y las políticas públicas tienden a asumir formas normativas y prescriptivas, intrusivas y homogeneizadoras. Los paradigmas que orientan las decisiones y acciones que desencadenan las intervenciones descansan en modelos teóricos simplificadores de la realidad social, cuyos supuestos de naturaleza causal, más que relacional, conducen a imponer modalidades de intervención que no toman en cuenta las preferencias y lógicas de operación de los agentes que se quiere intervenir. Es recurrente ver propuestas muchas veces ambiguas, con definiciones simples de los problemas y las estrategias de solución para llevarlas a cabo, asumidas de antemano como formulas o verdaderos axiomas por proponentes y ejecutores. En esta línea, las intervenciones y las políticas públicas suelen anclarse en visiones positivistas, en las que se parte del supuesto que lo social se puede observar, entender y manipular desde fuera. En esta perspectiva cuasi cibernética de la realidad, las modalidades de las políticas son prescriptivas y homogeneizadoras. A modo de ejemplo, un trabajo bastante difundido en los centros de estudio de las universidades latinoamericanas, que prescribe el camino para hacer políticas públicas a partir del modelo de los Estados Unidos de Norteamérica (Rushefsky, 2008), propone un conjunto de pasos que van desde la identificación de los problemas por parte del agente interventor, el 
diagnóstico, la formulación de la agenda, la dimensión presupuestaria, la implementación, y finalmente reformulación, con la misma lógica, sin considerar para nada las subjetividades y percepciones de los sujetos a ser intervenidos. Pero la identificación tanto como la definición de los problemas no debiesen limitarse a decisiones unilaterales de la autoridad o el sistema interventor, quien observa, diagnostica, evalúa y propone desde su propia lógica de operación. Los problemas debiense ser identificados, diagnosticados y definidos, primero, tomando en cuenta los códigos y símbolos de los sistemas sociales particulares que se desea intervenir, si la estrategia es trazada por un proponente, y, segundo, con alto grado de inclusión, comunicación, persuasión y participación, en la etapa de trabajo en la línea base y el diagnóstico del interventor con los agentes a ser intervenidos.

En línea con aproximaciones teóricas que cuentan con una base empírica consistente y que subrayan la importancia de la comunicación y la confianza en las regulaciones y las políticas públicas (por ejemplo Ostrom, 1990), en este estudio, argumentamos que las intervenciones sociales y las políticas públicas debiesen incluir modalidades que persuadan a que los intervenidos acepten la invitación que éstas proponen en sus propias formas de sentido, es decir, que lo que se seleccione, cómo se seleccione, qué se entienda, sea genuinamente resonante y transformador, y no un mero "ruido".

En esta línea argumentativa, a continuación, presentamos una reflexión sobre cómo la política pública se puede beneficiar con orientaciones que privilegien formas que inviten o persuadan a la transformación. En otras palabras, queremos conceptualizar con el énfasis puesto en la naturaleza compleja y dinámica de las relaciones sociales, la centralidad de la inclusión y de la comunicación, donde el proceso de intervención, en lugar de ser el convencimiento en torno a la implementación de una prescripción, es una suerte de invitación con alto grado de comunicación (Ostrom, 1990; Majone, 1997). En esta perspectiva, debemos comenzar por identificar anclajes teóricos que den sustento a una intervención comunicativa que parte del reconocimiento que cada sistema funciona con lógicas propias, es intransparente uno del otro, clausurado en sus operaciones, con un código único y una única función para dar cuenta de un problema para la sociedad.

\section{Desde la prescripción hacia políticas de persuasión}

En América Latina, convencionalmente, las políticas públicas han operado con una lógica de comando y control, en una sintonía de primer orden. En las intervenciones convencionales, podemos ver que hay un observador (interventor) que, desde fuera, trata de prescribir algo a un sujeto observado (intervenido), porque, según el primero, la aceptación de la propuesta tendrá resultados beneficiosos. En este juego, domina la vieja postura de un estímulo objetivo que determina a un sujeto, donde las políticas públicas, en cuanto intervenciones, se anclan en referentes epistemológicos que establecen una realidad fuera del sujeto y que éste tiene que aprehender. Es bajo esta lógica donde se sustentan y proponen generalmente las intervenciones en políticas públicas, donde un observador (proponente de la política) determina qué es bueno y pertinente para la superación de un problema - que él define como tal -, observado en la realidad. En otras palabras, nos encontramos con modelos simples que llamaremos de primer orden, que se alejan de modelos más complejos y reflexivos que podemos llamar de segundo orden.

Pero, en un contexto de las transformaciones sociales relacionadas con la globalización, democratización y el creciente pluralismo en todas las esferas de la vida humana, las sociedades latinoamericanas tienden a la automatización de sus sistemas (clausura de sus operaciones) y a la diferenciación de sus funciones, y ya no pueden operar con y desde centros que imaginen, prescriban y hagan, rol que han cumplido tradicionalmente los sistemas políticos centrales y sus 
políticas (Mascareño, 2000; Vallejos, 2007). No existe a priori una jerarquía u orden de importancia, porque cada sistema asume una posición prioritaria para producir y reproducir las operaciones de la sociedad. Las características de la sociedad moderna en que vivimos y sus sistemas funcionales nos llevan a plantear que, cuando cualquier órgano público o semipúblico trabaja en la elaboración de políticas públicas, deberá tener en cuenta el operar de una sociedad, que a un nivel organizativo despliega como forma primaria la diferenciación por funciones, pero a un nivel estructural sus formas tienden a ser plásticas.

Es del caso citar el planteamiento de Giandomenico Majone sobre la persuasión. Según Majone (1997), los modelos convencionales de políticas públicas han pasado por alto que los actores entran un continuo debate y dinámicas de persuasión, calibran sus visiones, y dan viabilidad a la política. Pero Majone hace notar que, hoy en día, la persuasión estaría siendo usada con un tinte racionalizador o de un uso deshonesto de los argumentos (propaganda, lavado de cerebro, manipulación). Ahora, si no fuera usada de esta forma, la persuasión sería un intercambio bilateral, un método de aprendizaje mutuo mediante el discurso, el que permitiría no sólo que los actores defendieran sus intereses y opiniones, sino que también ajustasen su visión de la realidad y pudieran cambiar sus valores. Para ilustrar lo dicho, queda muy claro el ejemplo que da Majone del físico que entrega un informe, cuya exposición es el esfuerzo de convencer de que el mundo se comporta tal como él lo ha concebido y no una descripción de lo ocurrido al realizar su investigación. Tras este descubrimiento, viene la demostración pública, el proceso deliberado de persuasión, donde el experimento es retóricamente una pieza poderosa para persuadir a la mente más obstinada y escéptica de aceptar una nueva idea. Puede esto ser una elección correcta, dice Majone, pero se necesita del argumento, la justificación, la explicación y la persuasión comunicativa.

La persuasión sería un arma con un potencial avasallante en la formulación de políticas pú- blicas. Un arma que permitiría, por medio de una argumentación racional, convencer a un público o auditorio de lo que se le está ofreciendo. En otras palabras, se apelaría al genuino consentimiento para aceptar una propuesta de intervención, que, según el proponente y el discernimiento autónomo del sujeto a ser intervenido, será efectiva para la solución de un problema. Si la persuasión fuese una invitación para la intervención, donde, concertada o unilateralmente, la propuesta de intervención se instalara en el entorno del intervenido como un regalo con sentido, y este último, entendiéndola así, la arrastrara a su interior, es decir, dando su consentimiento se apropiara de ella y la tomará, y este proceso condujera al cambio deseado, estaríamos ante una política pública no prescriptiva y con alta probabilidad de éxito.

Por lo tanto, en adelante, argumentaremos que una política pública como intervención no debe ser algo impuesto por el proponente, sino más bien debiese hacer uso de la persuasión como una invitación a aceptar la propuesta del interventor desde los códigos del sistema a intervenir, aumentando, así, la probabilidad de dar cuenta del problema que se desea solucionar.

\section{La política pública en el marco de una aproximación de segundo orden}

Los avances en las teorías del conocimiento han dejado atrás los enfoques positivistas que entendían la realidad biológica y social como un objeto que se puede explicar, manipular y determinar exógenamente. A modo de ilustración, en la línea de la fenomenología y el constructivismo, Humberto Maturana desarrolló una biología del conocimiento que parte de la constatación empírica de la imposibilidad de distinguir, en la experiencia, entre ilusión y percepción (Maturana, 1996, p. 30). Desde la Teoría de Sistemas, Niklaus Luhmann (1990) demostró que el observador y lo observado están determinados estructuralmente, que es su propia estructura y no algo externo lo que especifica su experimentar. Todo esto es también válido 
para los sistemas sociales, los que están condicionados de igual forma por las matrices que autoconstruyen. En el contexto esbozado, si nos inscribimos en los lineamientos que nos propone Luhmann, donde la sociedad estaría compuesta por comunicaciones, cada sistema o esfera social tiene un medio de comunicación especializado, que es clausurado y autónomo.

El observador es un observador de segundo orden, es decir, un observador que observa como observan los observadores. Por lo tanto, no son posibles las explicaciones que revelen algo independiente de las operaciones mediante las cuales se generan dichas explicaciones: la lógica de observación no puede sobrepasar la lógica del observador (sistema), donde la referencia de lo observado (descrito) siempre es el observador (sistema). Así, toda hetero-referencia es posible sólo como construcción del observador.

$\mathrm{Al}$ existir un alto grado de improbabilidad que la comunicación tenga éxito entre sistemas de las mismas características, resultan ser necesarias propuestas de políticas públicas que, en su forma de intervención, pongan énfasis en tres fases: selección de algo que se quiere comunicar (qué), la estrategia de comunicación (cómo) y si se entendió (comprensión). En otras palabras, el énfasis debe estar puesto en el intervenido y no en el interventor (intervención de segundo orden), dando paso a procesos auto-regulativos y no de control o prescripción. Una intervención informada en la propuesta descrita podría afrontar, de mejor manera, los cambios que se desean obtener para resolver problemas, conflictos o mejorar la calidad de vida de la población.

En el modelo de segundo orden, la lógica de intervención se invierte: la intervención se emprende desde el sistema del intervenido, asumiendo la estrategia en la forma del que se quiere transformar. La iniciativa de intervención no es intrusiva, ni directiva, ni normativa, sino que ésta se pone en el entorno del sistema a intervenir para que el intervenido la asuma como algo que le es propio y con sentido.

La atribución que emana desde el interventor debe estar adecuadamente informada de la unidad social donde se desarrolla la intervención, pues, en principio, la ceguera del observador ante la definición de un problema así como las estrategias para la solución de éste son naturales y propias de una sociedad moderna y diferenciada por funciones. De lo anterior se sigue que, cuando se quiere intervenir en sistemas que son autónomos o clausurados operacionalmente, lo adecuado será hacerlo no poniendo en peligro la autonomía de los sistemas intervenidos. La idea fundamental es que el sistema o grupo de actores que se intervenga pueda observar y entender las distinciones que ofrece el interventor (oferente de políticas) y procesarlas en su interior como información que tenga sentido. Con esto se quiere enfatizar la libre capacidad de los agentes a ser intervenidos para discernir y decidir por sí mismos cuál es el modo en que quieren operar. En este sentido, retomando los planteamientos de Majone, en una política pública razonable, tiene un papel central la persuasión, como una invitación (orientación) para que el propio sistema oriente sus operaciones en la dirección en que haya ordenado las distinciones del sistema interventor. En caso de ser resonantes dichas distinciones para el sistema a intervenir, es probable que éste último las introduzca en su operar y genere el o los cambios que el interventor desea obtener.

En el marco de una aproximación convergente, de priorizar al sujeto que será intervenido, David Easton (1965) subrayó que las dinámicas que alimentan el sistema político y sus decisiones proceden de la sociedad y sus inputs. En su conceptualización, el sistema político tiene por función la asignación autoritativa de ciertos valores, y es un sistema abierto, expuesto a las influencias derivadas de otros sistemas con los cuales interactúa. La lógica de relaciones del sistema político con su medio ambiente consiste en la transmisión de impulsos desde el medio y respuestas. Los impulsos son de dos tipos: demandas y apoyos. Las demandas son enunciadas dirigidos a las autoridades del sistema, que proponen algún tipo de asignación autoritativa. A 
su turno, la respuesta del sistema político genera un proceso de retroalimentación sobre el medio ambiente, que reacciona, procesando nuevas demandas y apoyos. Las demandas tienen su origen en intereses y deseos de grupos de personas que son canalizados hacia el sistema político por medio de la acción de entes convertidores, que pueden ser partidos políticos, grupos de presión u organizaciones sociales. Los apoyos son aquellos elementos que proporcionan estabilidad al sistema político.

\section{EXPERIENCIA CHILENA CON POLÍTICAS PÚBLICAS DE PRIMER ORDEN, EL CASO DE LATRASPARENCIAACTIVA}

\section{Características y génesis de la política chile- na con la transparencia}

La política chilena de transparencia ${ }^{1}$ representa un clásico ejemplo de una intervención de primer orden. La política consiste en la exigencia de medidas de mayor transparencia en los servicios públicos y los gobiernos municipales, por medio de disposiciones, regulaciones y la fijación de estándares sobre transparencia. La ley de Transparencia del año 2008 dispuso que los servicios y las organizaciones públicas deben mantener actualizados ciertos ítems de información en sus sitios web (transparencia activa), y deben responder consultas (transparencia pasiva). Adicionalmente, la ley creó un Consejo de la Transparencia con la función de monitorear la transparencia en el sector público.

En perspectiva histórica, el concepto de probidad fue introducido en el artículo 8 de la Constitución de 1980, en el cual se dispuso que los funcionarios públicos deben adherir a ese principio y que las acciones y decisiones del Estado deben ser públicas, excepto si un quórum calificado determinase que deben ser secretas. Bajo el gobierno del Presidente Eduardo Frei, la Ley de Probidad

1 Detalles y documentación sobre la génesis de la legislación, los proyectos y debates de los legisladores están disponibles en www.senado.cl
Nr. 19.653 (1999) estableció mecanismos para la publicación y el acceso a información pública. Adicionalmente, la legislación dispuso causas para negar el acceso a ciertas informaciones. Bajo el gobierno del Presidente Ricardo Lagos, la Ley 19.880 (2003) dispuso cómo publicar información sobre acciones administrativas con estándares de transparencia. Por otra parte, la legislación contenía cláusulas que daban la posibilidad de proteger información mediante decretos. Esas disposiciones podían operar a favor del secreto en los servicios públicos y fueron usadas para mantener información en reserva. El año 2005, entró una iniciativa de ley al Senado para modificar la legislación. El proyecto resultante, octubre del 2005, dispuso que los servicios fiscales debían hacer públicos ocho tipos de información: la estructura organizacional; los poderes de cada unidad administrativa; el listado de funcionarios contratados de planta, a contrata y a honorarios con los montos de sus salarios; los objetivos de cada unidad administrativa; las disposiciones legales y reglamentarias; el listado de pasos y requerimientos para acceder a beneficios; una descripción de los procedimientos para tener acceso a subsidios, incluyendo la lista de beneficiarios; y los mecanismos de participación ciudadana disponibles.

Durante la presidencia de la Presidenta Michelle Bachelet (2006-2010), el proyecto de ley ingresó a la Cámara de Diputados con indicaciones introducidas por el Ejecutivo. La nueva propuesta reforzó los principios de transparencia, especificó las restricciones para el acceso a información pública, incorporó el principio de la "transparencia activa", disposición de ciertos ítems de información en los sitios web, ${ }^{2} \mathrm{y}$ los procedimientos para tener acceso a información no publicada activamente.

${ }^{2}$ Los ítems son: actividades y documentos oficiales; marco regulatorio; esquema organizacional; personal por nombre, categoría y salario; adquisiciones mediante el Mercado Público y contratos; fondos públicos transferidos; actos $\mathrm{y}$ resoluciones; procedimientos y requerimientos municipales; requerimientos para acceder a subsidios y beneficios; mecanismos de participación; presupuesto y ejecución presupuestaria; auditorías; participación en corporaciones; banner de transparencia en el sitio web; formularios para requerir información; registro de actos y documentos; actos administrativos que disponen los precios para la reproducción de materiales. 
Además, dispuso sanciones por no publicar o proveer información y la creación de una agencia reguladora, el Consejo para la Transparencia. La ley 20.285 fue promulgada en agosto del 2008 y pasó a tener vigencia desde abril del 2009.

\section{Dificultades de la política en los gobiernos locales, los municipios}

El año 2011, un Monitoreo del Centro de Políticas Públicas de la Pontificia Universidad Católica de Chile, con ayuda de una escala sobre el cumplimiento de los ítems de la "transparencia activa” dispuestos por la Ley, detectó que, en 330 sitios web municipales, el nivel de cumplimiento con lo dispuesto en la Ley era del 30\% (CETIUC, 2011). Ningún municipio publicaba y actualizada información sobre los ítems, solo 1,8\% cumplía con 10 o más ítems, y el 60\% sólo entre 2 y 5 ítems. En abril del 2012, el Consejo de la Transparencia realizó una auditoria de las prácticas on line de cada municipio, con ayuda de una escala que consideraba 7 elementos para asignar puntuaciones a los municipios. En promedio, el puntaje alcanzado por los gobiernos municipales representó el 30,26\% de cumplimiento (Dirección de Fiscalización, 2012).

El Monitoreo observó que los municipios tenían información dispersa en distintos departamentos, carecían de apoyo tecnológico, equipamiento, personal y sistemas de manejo de información. Los municipios pequeños de zonas rurales tenían una puntuación más baja que el promedio, al igual que municipios donde más del $70 \%$ del presupuesto correspondía a recursos del Fondo Común Municipal (FCM). En suma, los municipios con mayores dificultades de desempeño con la "transparencia activa" eran aquellos con poca población, en áreas rurales, con poca autonomía presupuestaria. Sin embargo, había municipios con las características indicadas con alta puntuación.

En suma, la mayor parte de los municipios no estaban dando cumplimiento a lo prescrito por la ley, y quedó en evidencia que la política no había considerado numerosas condiciones y aspectos de las comunas y los propios municipios.

\section{La política de transparencia como intervención de primero orden, comando y control}

Una de las principales limitaciones de la política chilena de la transparencia activa es el enfoque de comando y control, con poco espacio para la persuasión, el diálogo y la facilitación. En esta concepción, el gobierno trata de ser más transparente por medio de legislación, implementación y hacer cumplir las leyes que prescriben que los servicios y las organizaciones públicas deben hacer pública la información. La política dispuso qué tipo de información debe proveer los municipios, sin considerar los contextos municipales ni las condiciones de los ciudadanos para poder exigir mayor transparencia. En una perspectiva crítica, desde una aproximación de segundo orden, podemos ilustrar la política de transparencia que fue impuesta a los municipios con ayuda del Figura 1.

En el esquema, observamos al subsistema municipal en relación con su medio ambiente, compuesto por otros subsistemas (partidos políticos, otros intereses corporativos, organizaciones sociales, población, gobierno), con los cuales interactúa, recibiendo estímulos, dando respuestas. Por otra parte, observamos la política de transparencia del Estado Chileno, consistente en la Ley de Transparencia, el Consejo para la Transparencia, y la transparencia activa (disponer cierta información en los sitios web) como una prescripción. En el esquema, observamos que la lógica de la política es de comando y control, no parte desde el subsistema municipal y los otros subsistemas integrados, por lo tanto pasa por alto numerosos factores, y su resultado es el mínimo cumplimiento por parte de los municipios. Al no ser una intervención de segundo orden, la política no puede ser integral, porque quedan fuera actores que no han sido persuadidos por la política, y, por lo mismo, tampoco puede ser facilitadora.

En la política chilena de la transparencia activa, la cantidad y el tipo de información 
Figura 1 - Modelo simplificado de sistema municipal de la transparencia

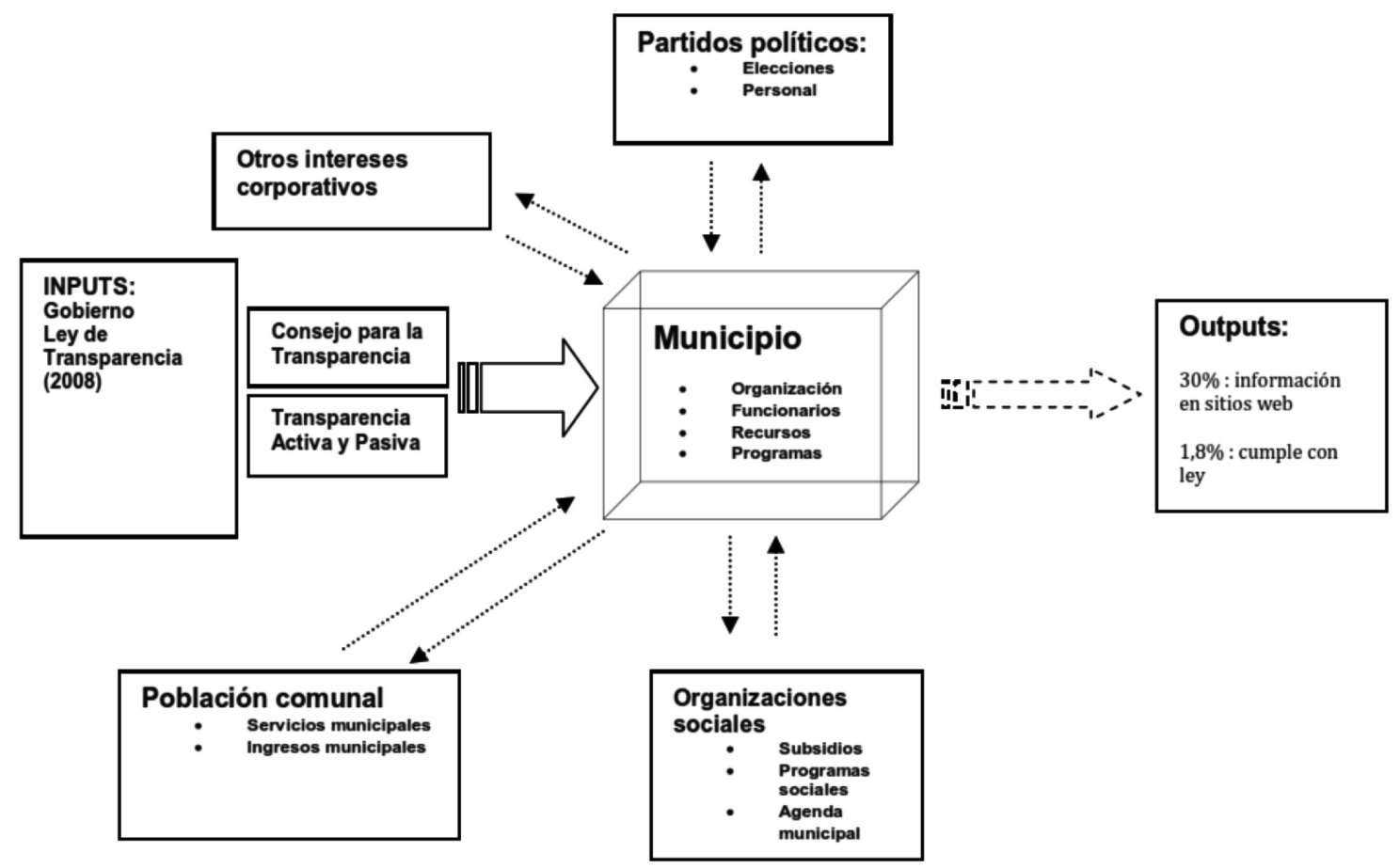

Fuente: Elaboración propia, con información de Valdivieso, 2012

dispuesta es lo fundamental. Pero la disposición de ese tipo de información no tiene efectos automáticos en la "evaluación de la información". Esa información no garantiza que los receptores potenciales prioricen o estén en condiciones de hacer uso de la información. Es difícil que lo estén, cuando hay factores que dificultan el uso que puedan dar las personas a ese tipo de información. Cabe tener presente que las personas de bajos ingresos, la mayoría de los chilenos, probablemente no cuenten con computadores o la posibilidad de conectarse a internet, que quienes tienen bajos niveles de educación probablemente no entiendan ese tipo de informaciones. Las debilidades de esta política quedan de manifiesto al considerar algunas características de la población chilena que, al parecer, no fueron tomadas en cuenta en la fase de diseño. A un año de la entrada en vigencia de la Ley de Transparencia, la encuesta de caracterización socioeconómica CASEN (2009) ${ }^{3}$ informaba que tan

${ }^{3}$ Aplicada a 246.924 personas, (disponible en http:// www.ministeriodesarrollosocial.gob.cl/ casen/ bases_datos.html) solo el 15,4\% de los encuestados contaba con educación media humanista completa, el 61\% no usaba computador, el 8,8\% disponía de computador en el hogar, el 4,1\% disponía de conexión a internet, y el 2,8\% usaba internet para tramites en instituciones públicas. Por otra parte, muy pocos conocían los mecanismos dispuestos para la comunicación entre las autoridades y los ciudadanos: las oficinas de información, reclamos, consultas y sugerencias eran conocidas por el $17,1 \%$ de los encuestados, las información de derechos ciudadanos en los servicios públicos por el 13\%, las cuentas públicas de la autoridad por el 14,8\%, los diálogos o diagnósticos participativos por el 8,6\%, los presupuestos participativos, plebiscitos o cabildos por el 11,9\%, los programas públicos participativos por el 10,9\%. En síntesis, las características educacionales, la disponibilidad de computadores y acceso a internet, y el grado de familiaridad de la población con instrumentos e informaciones relevantes para la transparencia indican que, probablemente, un muy bajo porcentaje de la población chilena se podría 
interesar y estaba en condiciones de participar en el mecanismo de transparencia dispuesto por los gobiernos chilenos.

\section{El contexto municipal chileno}

No obstante el papel central que tienen los gobiernos municipales para el desarrollo y su efecto en las condiciones de vida de la mayor parte de la población (Valdivieso, 2012), en buena parte de los municipios chilenos hay un conjunto de rigideces que dificultan la marcha de servicios y programas municipales eficaces y representativos, todo ello en perjuicio de la transparencia y la participación. Entre estos, cabe sintetizar algunos problemas recurrentes. ${ }^{4}$

En los municipios coexisten distintas visiones y comprensiones sobre la transparencia y la participación ciudadana, lo cual se traduce en diversidad y dispersión de criterios y formas de entender el concepto y aplicar los instrumentos disponibles, entre ellos las disposiciones de la Ley de Transparencia. En dos municipios distintos, transparencia puede llegar a ser entendida en sentido distinto por las autoridades políticas; para un Alcalde, ella puede consistir en reunir gente para que escuche su cuenta o discurso, mientras que, para otro, puede ser entendida como reunir a dirigentes de las juntas de vecinos para informarles sobre proceso de diseño del plan de desarrollo comunal. La diversidad y la dispersión de criterios explica una enorme variedad de iniciativas programáticas eintervenciones que se describen como "transparentes", sin llegar a ser claro en qué sentido lo son, qué instrumentos de medición incorporan, y siéstos son adecuados para incentivar la participación de los ciudadanos y de sus organizaciones en la política de transparencia, en las decisiones locales y en los espacios de gobernanza. Por ejemplo, los Planes de Desarrollo Comunal (PALDECO), que según

${ }^{4}$ Información proporcionada por funcionarios de la SUBDERE, de la División de Organizaciones Sociales de la Secretaría General de Gobierno, y funcionarios de diversas municipalidades, en ocho talleres y por medio de entrevistas (talleres entre diciembre 2006 a noviembre del 2007; entrevistas durante el año 2012). la ley debiesen ser instrumentos de planificación y desarrollo con alto grado de participación social, son diseñados por empresas consultoras que priorizan los temas de interés en los programas de gobierno y para las autoridades municipales.

La mayor parte de los municipios no hace uso de instrumentos y mecanismos que colaboren con la sintonía real y regular entre los programas municipales y las preferencias y posibilidades concretas por transparencia y participación de los ciudadanos, según sus contextos socioeconómicos, culturales y sus características territoriales específicas. Los sondeos de las encuestas indican que los ciudadanos tienen muy poco contacto con los municipios, y la participación en instancias dispuestas para la fiscalización es extremadamente baja; en la encuesta LAPOP 2010 (disponibles en http://www.vanderbilt.edu/lapop/), el 77\% de los encuestados informó no haber realizado trámites en su municipio durante los últimos 12 meses, y solo el 4\% declaró haber asistido a una sesión municipal o un cabildo abierto.

La falta de esos vasos comunicantes y espacios municipales sustentables de participación se combina con numerosas restricciones en términos de facultades, atribuciones y presupuesto, $\mathrm{y}$ la multiplicación de programas e iniciativas asociadas a políticas del gobierno central y sus servicios centralizados, cuyos objetivos son exógenos, y cuya duración depende de decisiones que escapan al rango de facultades y atribuciones de los funcionarios municipales.

En las comunas, los vecinos casi no intervienen en el presupuesto municipal, uno de los principales instrumentos de gestión y transparencia. Los presupuestos participativos, si bien han sido introducidos en algunas comunas, están limitados a un pequeño porcentaje de recursos de inversión, quedando la mayor parte del presupuesto municipal fuera de la consideración de los ciudadanos. Por lo tanto, la posibilidad ciudadana de informarse e influir en las decisiones municipales está altamente limitada.

En síntesis, los municipios chilenos tienen numerosas condiciones adversas para fortalecer la 
participación social y la transparencia. Sin embargo, la política de transparencia ha priorizado sólo un aspecto: disponer de información en los sitios web, sin considerar numerosas condiciones, probablemente por ser el más fácil de monitorear tanto dentro como fuera de Chile.

\section{Cultura política, partidos, organizaciones sociales y transparencia}

Las últimas elecciones chilenas del año 2012, con un 60\% de abstención electoral, sugieren que un sector significativo de la población de las comunas se mantiene al margen de la política local, y, probablemente, no canaliza inquietudes en el ámbito municipal. Por otra parte, la participación social en asociaciones y la confianza social es baja. En el campo de las actitudes y percepciones, la mayor parte de los chilenos no tiene interés por los asuntos públicos y la participación política es baja. La encuestas del Proyecto LAPOP, aplicadas los años 2010 y 2012 (disponibles en http:// www.vanderbilt.edu/lapop/), informan que más del 70\% de los encuestados tiene poco o ningún interés por la política. Aproximadamente el $52 \%$ de ellos considera que no entiende bien los asuntos políticos más importantes del país. La encuesta del Barómetro Regional del año 2011(disponible en http://politicaspublicas.ulagos.cl/barometro-regional/), al consultar si las personas conocen como funcionan las principales institucionales nacionales, regionales y comunales, informa que, en promedio, más del 70\% de los encuestados responde que no. Más allá de saber quien ocupa el cargo de la presidencia de la República y conocer otras personalidades gracias a la cobertura de los medios, las personas no disponen de información ni entienden cuales son las funciones de las principales autoridades del ejecutivo, del legislativo, los gobiernos regionales y locales.

Las mismas fuentes informan que la mayor parte de las personas encuestadas se siente satisfecha y valora su sistema democrático, respetan sus instituciones, perciben que, en el país, hay consideración por sus derechos, no tienen percepción de problemas de corrupción, y confían en sus municipios y en sus alcaldes. En promedio, para los años 2010 y 2012, más del 80\% de los consultados por la encuesta LAPOP consideró a la democracia como la mejor forma de gobierno, sobre el $67 \%$ informó tener respeto por las instituciones políticas chilenas, el $58 \%$ estimó que los derechos de los ciudadanos están bien resguardados, el $65 \%$ declaró tener confianza en el municipio. Paradojalmente, la misma fuente informa que más del $77 \%$ de esas personas no tiene experiencias recientes de trámites en el municipio y solo el 4\% recuerda haber asistido a una sesión municipal o un cabildo abierto.

Cuando las personas declaran estar satisfechas con la democracia, consideran que Chile es un país democrático y respetan las instituciones, sin mucha experiencia, información ni interés por entender complicados procedimientos y asumir altos costos de tiempo y recursos para informarse, es altamente probable que estén conformes con la estructura que tiene el estado, las funciones formales del gobierno municipal, y por lo tanto resulta poco probable que vayan a exigir esfuerzos especiales por parte del municipio por incrementar los niveles de transparencia, en particular el tipo de información de la "transparencia activa". Por el contrario, probablemente si las personas no estuviesen satisfechas con la democracia tendrían mayor predisposición a manifestar actitudes críticas con respecto al municipio y exigir más información.

Los partidos políticos chilenos tienen estructuras centralizadas, dominadas por las cúpulas y directivas, disponen de poco espacio para la representación y participación de las bases y de los intereses territoriales (Joignant, 2012). Por otra parte, por las disposiciones del sistema electoral chileno, los alcaldes son elegidos cada cuatro años por medio de un sistema electoral mayoritario, y los concejales por medio de un sistema proporcional, que garantiza representación a las dos grandes coaliciones políticas del país, la centro izquierda Concertación de Partidos por la Democracia y la centro derecha Alianza por Chile. La mayor parte 
de los municipios son gobernados por alcaldes de ambas coaliciones, y sus concejos municipales tienen representantes de ambas coaliciones. La tasa de reelección es alta, con alcaldes y concejales que mantienen sus cargos durante varios períodos (información disponible en www.servel.cl). Por lo tanto, los partidos tienen asegurada su participación en el gobierno y en las políticas del municipio, y no manifiestan especial preocupación por la transparencia en sus programas.

En tales condiciones, es poco probable que los partidos cumplan la función de canalizar demandas sociales por transparencia hacia los municipios, y lainformación disponible indica poca identificación social con los partidos. Los sondeos de opinión informan que existe un gran distanciamiento entre la sociedad y los partidos políticos, y la confianza en ellos y en los políticos es baja. En la encuesta LAPOP 2012, en una escala de 7 puntos, el 70,1\% de los encuestados manifestaban poca o ninguna confianza en los partidos, el 50\% consideraban que la democracia podría existir sin partidos, y tan solo el 1,4\% que, en caso de tener algún conflicto con el municipio, recurría a un familiar o a un conocido con contactos políticos.

Por lo tanto, los partidos políticos tienen intereses y están involucrados con los intereses de los gobiernos municipales, y no representan a importantes sectores sociales en las comunas, y probablemente no tienen incentivos para canalizar inquietudes sociales por transparencia.

Por otra parte, el asociativismo social en las comunas chilenas tiene un modesto desarrollo, $\mathrm{y}$ las organizaciones canalizan un mínimo de inquietudes sociales hacia los municipios. Según informan las encuestas del PND (disponibles en www.pnud.cl), la vida asociativa y la confianza social en Chile son relativamente bajas, si se compara con otros países del mundo (Frane, 2008). En las comunas, las organizaciones territoriales y funcionales (juntas de vecinos, organizaciones del adulto mayor, comités comunitarios, centros de padres, etc.) postulan a subsidios municipales y canalizan información, tienen sus reuniones mensualmente, $\mathrm{y}$ los dirigentes tienen un rol cen- tral en la dirección y el control. Ellas operan en relación con objetivos muy puntuales, proyectos específicos, y, por lo general, no tienen una vida cívica autónoma. Además tienden a estar encapsuladas, disponen de pocos vínculos con otras organizaciones, y, por lo tanto, desarrollan modestos niveles de capita bridging o puente. A su turno, los municipios promueven este tipo de organizaciones, por cuanto ellas son funcionales a sus propósitos y políticas.

Las organizaciones sociales, carentes de recursos y capacidades autónomas, dependientes de los subsidios y programas sociales del municipio, difícilmente estarían en condiciones de canalizar inquietudes y presiones por transparencia. Por el contrario, sus dirigentes tienden a manejar las relaciones con el municipio, y son quienes controlan los flujos de información. Por lo tanto, resulta ser razonable esperar que la participación en esas organizaciones, en lugar de promover la canalización de inquietudes sociales y ciudadanas por transparencia, tenga el efecto contrario. Es decir, a mayor cantidad de vida asociativa, mayor grado de conformismo con el municipio, y menor la demanda por transparencia. En consecuencia, menores los incentivos del municipio por mejorar su transparencia activa.

En síntesis, todas estas informaciones dan cuenta de un contexto social, cultural y político que tiene claros efectos en las dinámicas sociales y los inputs por transparencia, y, sin embargo, está al margen de la política chilena de la transparencia.

\section{La percepción de los funcionarios municipales}

La aprobación de leyes de transparencia puede manifestar la preocupación de los gobiernos por el manejo democrático de la información, pero, cómo esto se lleva a cabo e impacta en las prácticas democráticas, depende de aquellos que trabajan con la información y la transparencia día a día. Por esta razón, los niveles de desempeño municipal pueden variar debido a características, percepciones, valoraciones y los contextos 
organizacionales que influyen en las decisiones y acciones de los funcionarios. En una política pública de segundo orden, el diálogo con los funcionarios públicos encargados de aplicar la política debiese ser una prioridad.

Con la orientación de estudios que han analizado la perspectiva de los funcionarios públicos sobre las políticas de transparencia (Fairbanks et al. 2007), procedimos a elaborar un cuestionario para explorar las percepciones de los funcionarios municipales chilenos. El cuestionario incorporó 45 preguntas sobre temas que han sido identificados como relevantes por otros estudios: entendimiento de la transparencia, valor de las comunicaciones, posición de las autoridades, aspectos de la organización, relaciones con los medios de comunicación, entre otros. El cuestionario fue enviado a 52 municipios en la Región Metropolitana de Chile, y 21 cuestionarios fueron completados y retirados de las oficinas municipales. ${ }^{5}$

Una pregunta destinada a conocer el grado de acuerdo de los funcionarios municipales con la intervención del gobiernoen las decisiones municipales deja en evidencia que hay resistencia a una política de comandoy control:el 90\%delos consultados consideró que el gobierno no debe decidir hasta que nivel el municipio debe ser transparente.

En una mirada agregada, las respuestas indican que hay percepciones que podrían tener efectos negativos en las decisiones de una parte de los funcionarios al trabajar en actividades de transparencia. Más de la mitad de los consultados no se percibe a sí mismo como un vínculo importante entre el público votante y el gobierno local, y un quinto piensa que apoyar la transparencia no ayudará al público a tomar decisiones informadas. Adicionalmente, un poco más de la mitad de los consultados considera que ciertas informaciones no debiesen ser dispuestas al público. En el marco de una aproximación más integral de la políti-

${ }^{5}$ Por un compromiso de confidencialidad, no es posible revelar qué municipios respondieron el cuestionario. Pero podemos informar que se trata de 22 municipios representativos de la Región Metropolitana, donde vive más del $70 \%$ de la población chilena. ca, el mayor conocimiento sobre las percepciones de los funcionarios y el esfuerzo con ellos para aclarar mejor su propia posición, podrían ser beneficiosos para alcanzar las metas de la política. En el diálogo entre la organización y el público, los medios y la prensa local pueden facilitar el intercambio de información. Pero solo el 38\% de los funcionarios municipales trabaja con la prensa local o con el público para un mejor entendimiento de los instrumentos de transparencia. Con todo, más de la mitad de los consultados (57\%) considera que habría que destinar tiempo para programas que faciliten el mejor entendimiento de los medios e instrumentos de la transparencia, y la mayoría (81\%) considera que ello representaría un buen uso del dinero. En este sentido, una política más persuativa, de diálogo y facilitación, de trabajo conjunto con los municipios, en programas que informen a los ciudadanos sobre cómo usar los instrumentos disponibles para la transparencia, sería una buena estrategia para corregir la política.

En la misma línea, ayudaría el trabajo en la comunicación interna de las organizaciones. Los funcionarios valoran positivamente recibir información del alcalde y del equipo directivo; el $62 \%$ cree que estar presente cuando se toman las decisiones ayudaría a comunicar mejor la política. Pero solo 48\% tiene encuentros con el alcalde o participan en reuniones donde se toman decisiones. El recurso más valorado es el personal; el $57 \%$ de los consultados piensa que se podría ser más efectivo con más gente. Reevaluando como se asignan las tareas de la transparencia en los municipios o racionalizando procesos y siendo creativos para movilizar mas unidades trabajando en la transparencia podría ayudar a mejorar la experiencia en general.

\section{CONCLUSIONES YORIENTACIONES}

Las dificultades de implementación de la política chilena de transparencia en los municipios indica que es necesario el esfuerzo por revisar esta política. Hemos identificado algunos de los pro- 
blemas que Chile ha tenido con la transparencia, principalmente la falta de una aproximación de segundo orden, una intervención que entienda la transparencia como un sistema interactivo, y la política más como un canal facilitador y de diálogo. Es particularmente importante considerar las percepciones y preferencias de quienes son responsables de la implementación de la política. En los municipios, esas personas tienen una posición central porque son los únicos que organizan y presentan la información al público a través de la "transparencia activa” y la "transparencia pasiva”, y probablemente por otros medios. Por lo tanto, tiene sentido dirigirse a ellos para entender mejor cómo asumen su responsabilidad. Es necesario clarificar y reforzar la misión de la transparencia municipal, fortalecer la cooperación inter-municipal, las interacciones entre el municipio y sus vecinos y entre el municipio y el gobierno central, en una suerte de diálogo de dos bandas, mejorar las comunicaciones internas, facilitar procesos, y fomentar el apoyo de la transparencia en el nivel de la gestión.

En sociedades altamente diferenciadas y complejas, la estrategias de intervención de las políticas públicas debieran: 1) distanciarse de modelos simples que conducen a intervenciones intrusivas en las operaciones del sistema a regular, pues intervenciones de esa naturaleza inducen dinámicas de operación en otra sintonía o distinta frecuencia, tal como se observa en el caso de la transparencia en los municipios; 2) expresar una genuina invitación que tenga en cuenta que todo sistema está clausurado en términos operacionales (códigos), pero abierto a la información del entorno; 3) priorizar, en sus formatos de aproximación, la comunicación y la persuasión, distanciándose de modalidades de interferencias intrusivas y disruptivas, desde la perspectiva de las operaciones del sistema a regular.

Cabe subrayar que toda política pública deberá propiciar que el propio sistema a ser afectado reconozca, en sus distinciones, aquello que la intervención pretende hacerle ver. Es decir, se trata de que el cambio a ser introducido no aparezca como una intervención exógena, una variable externa, ajena al sistema al cual está dirigido. El papel de la intervención de segundo orden consiste en hacer que el propio sistema procese el cambio como necesario para una operación óptima o simplemente para seguir funcionando. Aquí el concepto de resonancia, en lugar de "ruido" (Luhmann, 1996a), alude al proceso que dará sentido a lo expuesto, es decir, la coordinación de los significados de la intervención con los significados internos en el sistema.

Recebido para publicação em 29 de agosto de 2013 Aceito em 12 de janeiro de 2014

\section{REFERENCIAS}

BANCO MUNDIAL. Una nueva geografía económica. Washington D.C.: Banco Mundial, 2009.

CETIUC. Consultoría para el desarrollo del modelo de gestión de transparencia para municipios, en http:// www.consejotransparencia.cl/cplt-presenta-primerestudio-sobre-transparencia-municipal/consejo/2011-0823/180348.html [12 de agosto de 2013].

DIRECCION DE FISCALIZACION. Fiscalización en el sector municipal sobre transparencia activa, en http:/ www.consejotransparencia.cl/fiscalizacion-en-el-sectormunicipal-sobre-transparencia-activa/consejo/2012-0427/111106.html [12 de agosto de 2013].

EASTON, David. A systems analysis of political life. New York: John Wiley and Sons, Inc., 1965.

FAIRBANKS, Jenille et al. Transparency in government communications. Journal of Public Affairs, v. 7, n. 1, p. 23-37, 2007.

FRANE, Adam. Mapping Social Capital Across Europe: Findings, Trends and Methodological Shortcomings of Cross-National Surveys". Social science information. v. 47, n. 2, p. 159-186, 2008.

FLORINI, Ann. Increasing Transparency in Government. International Journal on World Peace, v. 19, n. 3, p. 3-37, 2002.

HEISE, J. A. Toward Closing the Confidence Gap: An Alternative Approach to Communication between Public and Government. Public Affairs Quarterly. v. 9, n. 2, p. 196-217, 1985

JOIGNANT, Alfredo. ¿Descentralización functional o territorial? Elementos de sociología de los agentes políticos en tres regiones de Chile (1989-2009), en: Delamaza et al. Nueva agenda de descentralización en Chile. Sentando mas actores a la mesa. Santiago: RIL Editores, p. 511-558, 2012.

LUHMANN, Niklas. Die Wissenschaft der Gesellschaft. Frankfurt am Main, Suhrkamp, 1990.

Die Gesellschaft der Gesellschaft. Frankfurt am Main, Suhrkamp, 1997.

MAJONE, Giadomenico. Evidencia, argumentación y 
persuasión en la formulación de políticas. México: Fondo de Cultura Económica/Colegio Nacional de Ciencias Políticas y Administración Pública, A.C., 1997.

MATURANA, Humberto. La realidad: ¿Objetiva o construida? II. México: Anthropos/Universidad Iberoamericana/Iteso, 1996.

MASCAREÑO, Aldo. Diferenciación funcional en América Latina: los contornos de una sociedad concéntrica y los dilemas de su transformación. Revista Persona y Sociedad, v. 14, n. 1, p. 187-207, 2000.

OECD. Better Life Index - Chile, en http:// www.oecdbetterlifeindex.org/countries/chile/ [12 de agosto de 2013].

Checklist for e-Government Leaders, en www.oecd.org/dataoecd/62/58/11923031.pdf [12 de julio de 2013]

Ethics in the public service. Public management occasional papers. n. 14, Paris, 1996.

Building public trust: ethics measures in OECD countries. PUMA Policy Brief, n. 7, Paris 2000.

OSTROM, Elionor. Governing the commons. Cambridge University Press, 2000.
PIOTROWSKI, Suzzanne et al. An Analytic Framework for Open Meetings and Transparency". Public Administration and Management. v. 15, n. 1, p. 138-176, 2010.

RAWLINS, Brad. Give the Emperor a Mirror: toward developing a stakeholder measurement of organizational Transparency. Journal of Public Relations Research, v. 21, n. 1, p. 71-99, 2009.

RUSHEFSKY, Mark. Public Policy in the United States. New York City: M.E. Sharpe, Inc., 2008.

VALDIVIESO, Patricio. Estado e Integración: Movimientos de Larga Duración, Estado y Preguntas de Investigación”. Revista de Ciencia Política. v. 22, n. 2, p. 85-104, 2002.

State and national economy in Latin America, en R. Stemplowski (ed.). On the State of Latin American Nation-States. Approaching the Bicentenary. Kraków: Andrzej Frycz Modrzewski Kraków University College Press, p. 85-135, 2009

Participation, associations and trust, implications, case of Chile. Bulletin of Latín American Research. v. 31, n. 3, p. 336-35, 2012.

VALLEJOS, Arturo. La política en la periferia de la modernidad. Esbozos para la observación en América Latina. Revista Cuadernos del CENDES. n. 65, p. 95-128, 2007. 


\section{FIRST ORDER POLITICAL INTERVENTIONS: the case of Chilean transparency policy}

\author{
Patricio Valdivieso \\ Arturo Vallejos-Romero
}

This study evaluates and interprets the difficulties found in the Chilean policy for transparency in municipalities, using the theory of social systems. This theory argues that, in Latin America's current context, public policies should respond to a society increasingly complex and diversified, with ever greater proximity to persuasion, dialog, and facilitation. In this case, the transparency policy, in terms of a first order intervention, has been a command-and-control policy, the reformulation of which should consider what this policy considers as transparency both for citizens and municipal employees.

KeY wORDs: Public policies. First order intervention. Transparency.

\section{INTERVENTIONS POLITIQUES DE PREMIER ORDRE: Le cas de la politique chilienne de la transparence}

\author{
Patricio Valdivieso \\ Arturo Vallejos-Romero
}

Cette étude évalue et interprète, en utilisant la théorie des systèmes sociaux, les difficultés auxquelles la politique chilienne est confrontée dans le cadre de la transparence des municipalités. Cette théorie soutient que dans le contexte latinoaméricain actuel les politiques publiques se doivent de répondre à une société de plus en plus complexe et diversifiée et se rapprochent de la persuasion, du dialogue et de la facilitation. Dans le cas présent, la politique de transparence, en termes d'intervention de premier ordre, a consisté en une politique de commande et de contrôle, et sa reformulation doit prendre en considération ce qu'elle entend par transparence pour les citoyens et pour les fonctionnaires municipaux.

Mots-CLÉs: Politiques Publiques, Intervention de deuxième degré, transparence.

Patricio Valdivieso - Doctor Philosopie pela Kahtolische Universitatet Eichstaett, Alemania. Profesor Titular del Centro de Estudios de la Realidad Local y Regional de la Universidad de los Lagos. Asociado a ORPAS, Universidad Bernardo O’Higgins. Coordinador del Area Estudio de las Dinámicas Sociales y Políticas del Territorio. Sus áreas de investigación son: ciência política y políticas publicas, gobernanza medioambiental, desarrollando investigación como Investigador Responsable del Proyecto FONDECYT/CONICYT Nro. 1140672 “Gobernanza Policéntrica y Capital Social puente/bridging como factores explicativos de la capacidad de respuesta y adaptación de los sistemas de gobernanza local ante los efectos del cambio climático y eventos extremos en Chile " (2014-2018). Algunas de sus más recientes publicaciones son: Opening the black box of social capital formation. American Political Science Research. 108 (1) 2014; Participation, associations and trust, implications, case of Chile. Bulletin of Latín American Research (ISI). 31 (3), p. 336-351, 2012; Capital Social, decisiones, factores políticos e institucionales. Casos: Chile y Uruguay. Revista de Ciencias Sociales. 18 (2), 204-215, 2012

Arturo Vallejos-Romero - Doutor en Ciencias Sociales por la Facultad Latinoamericana de Ciencias Sociales (FLACSO) México. Professor e investigador del Núcleo Científico Tecnológico en Ciencias Sociales y Humanidades de la Universidad de la Frontera, Temuco, Chile. Desenvolve pesquisas na área de sociologia del riesgo, intervención socioambiental, Gobernanza, políticas públicas y teoria social. Publicaciones recientes: Salmonicultura 2.0 en Chile: una mirada desde la gobernanza ambiental. Revista Venezolana de Gerencia (Scopus), Año 19, n. 65, enero-marzo, p. 116-137, 2014; Comunicación de riesgos ecológicos. El caso de la contaminación atmosférica en dos ciudades intermedias del sur de Chile. Revista Internacional de Contaminación Ambiental (ISI), v. 29, n. 1, Enero-Marzo, México, p. 59-75, 2013; Los déficits de calidad de la democracia en América Latina. Revista de Ciencias Sociales (ISI), v. 18, n. 2, abril-junio, p. 187-203, 2012; La relevancia de la confianza institucional y la comunicación en la percepción y construcción social de riesgos. Revista Perfiles Latinoamericanos (ISI), n. 39, enero-junio, p. 151-176, 2012. 